Z. vergl. Physiologie 73, 249-273 (1971)

(C) by Springer-Verlag 1971

\title{
Receptor Organization and Function in Limulus Chelae
}

\author{
Gordon A. Wyse \\ Department of Zoology, The University of Michigan, Ann Arbor, Michigan
}

Received April 7, 1971

Summary. 1. The opposed grasping surfaces of the digits of the chela of the Limulus walking leg have ridge-like pads of pliable euticle. Receptors are present both on these pads and elsewhere on the surface of the chelae.

2. Mechanoreceptors at the pad are rapidly adapting (Type 1) or slowly adapting (Type 2) (Fig. 1). Both types have thresholds of between 3 and $40 \mathrm{~g}$ and receptive fields of $1.2-2.5 \mathrm{~mm}$ along the pad (Fig. 3). Response frequency of Type 1 units increases with increasing rate of force change, while the number of spikes increases with increasing force magnitude (Fig. 4, 5). Response frequency and response duration of Type 2 units increase with increasing amplitude of force, but with some movement sensitivity (Fig. 7). Evidence is presented that the receptors involved are the large multipolar cells under the pad and that they have large rapidly conducting axons representing the fast sensory component of the large leg nerve.

3. Chemoreceptor units respond to clam or fish extracts or to glycine, glutamic acid, betaine, or trimethylamine oxide (Fig. 11). Most are tonic, but some units adapt completely within a few seconds, even to constant-flow stimulation. The tonic chemoreceptors are located at the small channel sensilla of the pad and perhaps also of the sides. Their axons are probably the smallest and slowest in the leg nerve.

4. The behavioral response to chemical stimulation of the chelae of intact animals is opening of the stimulated chela (Fig. 12).

5. Other sensory units give phasic or tonic responses to warm or cold sea water, or tonic responses to distilled water and to dilute sea water. The units are inferred to have axons intermediate in size between mechanoreceptors and chemoreceptors, the size decreasing in the order listed.

6. A proprioceptive organ of the tibiotarsal joint contains units responding to closing movement and units responding to closed position. It lacks units responding to opening or to open position.

7. The variety of claw receptors and the large number of sensory cells $(300000$ 400000 estimated per claw or 3-4 million total) indicate that the claw sensory apparatus represents a major sensory input into the central nervous system of Limulus. An estimated total of nearly 4 million chemoreceptors (3 million from claws, 1 million from gnathobases) may relate to the very large and well developed corpora pedunculata of the Limulus brain.

\section{Introduction}

The present study is an attempt to characterize the chelae of Limulus walking legs in terms of the types of receptors present, the manner of sensory coding, and the channels for transmission of information to the 
central nervous system. Limulus is considered to be a relatively unspecialized member of a subclass of primitive arthropods (Xiphosura). Therefore as suggested by Hodgson (1965), information about Limulus receptors may contribute to an understanding of the sensory organization of primitive arthropods and the evolution of arthropod receptor cells. Limulus is a bottom-dwelling, primarily burrowing animal. The chelae of the walking legs are used in feeding as well as in locomotion. Worms and molluses in the sand are picked up by the chelae and passed to the chelicerae and gnathobase region of the coxae for maceration. In view of their importance in feeding and locomotion the chelae probably represent a major part of the exteroceptive apparatus of the animal.

The sensory structures of the chelae are similar to those of the gnathobase spines of the coxae (Patten, 1893; Hayes, 1966; Wyse, 1967). There are two types of sensilla, simple channels (channel sensilla) and channels ending in pegs (peg sensilla). The fine structure of channel sensilla of the chilaria and flabellum, recently described by Hayes (1971), is quite similar to that of channel sensilla of the chelae.

Studies of receptor function in Limulus legs have concentrated on the proximal portions of the legs. Receptors on the gnathobase spines respond to chemical. thermal, mechanical. and water stimulation (Barber, 1956, $1961 \mathrm{a}, \mathrm{b}$; Barber and Hayes, 1963). Proprioceptors are present at each of the joints (for review see Hayes and Barber, 1967). The function of receptors of the chelae has been examined only in passing by Patten (1893), who found evidence of chemical and thermal sensitivity.

In the present study the physiology and certain aspects of behavior associated with the receptors of the chelae were examined. Characteristic and repeatable responses were found to mechanical, chemical, osmotic, thermal, and proprioceptive stimulation of the claw. Most but not all units appeared to be modality specific within the range of stimuli tested. Thus a fairly wide variety of physiological receptor types was found, in contrast to only a few apparently simple kinds of sensory structures.

\section{Materials and Methods}

Adult (18-24 cm maximum width of cephalothorax) and immature $(5-9 \mathrm{~cm})$ Limulus polyphemus (L.) were obtained from the Marine Biological Laboratory, Woods Hole, Massachusetts, and were maintained in a 200-gal recirculating sea water system. Most of the animals were used within a month after arrival, and all within four months.

Electrophysiological Recording. Recordings were made from isolated legs; usually several legs from the same animal were used in succession. The leg was cut off at the proximal end of the patella and the large and small leg nerves were exposed, generally by cutting through the skeleton and muscles at the patello-tibial joint and slowly pulling the two segments apart. The nerves were pulled out of the patella and usually were desheathed in the process. When care was taken to minimize stretch- 
ing the nerves, this procedure gave better results than exposing the nerves by dissection. The chela was fastened to a paraffin block, and the nerves, kept in artificial sea water, were subdivided with fine tungsten needles and watchmakers forceps. Fine nerve strands were lifted onto silver or platinum wire electrodes. The nerve impulses were amplified and displayed by conventional means.

For recording conduction velocities of mechanoreceptive fibers, the chela was stimulated by hand and the resulting action potentials were recorded at two locations on the attached nerve by separate pairs of electrodes within a moist chamber.

Mechanical Stimulation. Stimuli were applied by one of three methods. For qualitative studies the chela was stimulated by hand with a small probe. For quantitative studies defined stimuli were given by a stylus attached to the moving coil of a small loudspeaker. The coil was driven by a battery and switch or by a Grass S4 stimulator. Threshold and mapping experiments were conducted with a plastic probe screwed to a Grass FT10 force transducer. The output of the transducer was amplified and displayed on one beam of the oscilloscope. The transducer was clamped to an Emerson micromanipulator, the vertical movement of which was controlled by hand. Although this system allowed only limited control of stimulus waveform, the stimulus was accurately monitored by the transducer.

Either the loudspeaker assembly or the Emerson manipulator was mounted on a heavy movable-stage base plate. The locus of stimulation of the chela was varied by means of controls on the base plate. The chela was set in dental impression plaster to provide support and prohibit movement.

Fluid Stimulation. Fluid stimuli were applied in either of two ways. For determining qualitative responses of the receptors, the chela was held in air and solutions applied with a dropping pipette. The solutions were prevented from directly stimulating the exposed nerve by a rubber dam partition through which the chela passed, and by the continuous counterflow of sea water perfusion from the nerve toward the chela. The effectiveness of the barrier was confirmed at the end of some experiments by applying methylene blue solution to the chela and observing that it did not cross to the nerves.

To achieve defined fluid stimulation, a chamber was used in which part of the chela remained immersed. Either the tarsus or the index of the tibia was pushed through a pinhole in a rubber dam into the lumen of a closed Plexiglas chamber. A fluid switch allowed one of three stimulating solutions or sea water (for controls and for washing) to enter the chamber through an inlet tube. Placement of the outlet tube at the top of the chamber kept the chamber full at all times.

The rate of fluid exchange in the above apparatus was determined in separate experiments by alternately switching a weak ink suspension and water through the chamber and measuring with a photocell the intensity of light transmitted through the chamber. Under experimental conditions there was no solution exchange for the first 0.3 sec after switching; there was $50 \%$ exchange at 1.2 sec and $95 \%$ exchange at $4 \mathrm{sec}$. The following fluids were used to stimulate the chela receptors: decanted or filtered suspensions of ground fish (frozen ocean perch) or clam (fresh Mercenaria mercenaria) in sea water, $0.01-0.1 \mathrm{ML}$ betaine $\mathrm{HCl}, 0.01-0.1 \mathrm{M}$ trimethylamine oxide (TMO), 0.1-0.2 M glycine, $0.01 \mathrm{M}$ or saturated solution of glutamic acid (all made up in sea water); sea water at $\mathrm{pH} 2-3$ or $9-10$ (adjusted with $\mathrm{HCl}, \mathrm{H}_{2} \mathrm{SO}_{4}$ or $\mathrm{NaOH}$ ), distilled water, tap water, $40 \%, 60 \%$, and $80 \%$ sea water in distilled water, $40 \%$ sea water made isotonic with sucrose, and sea water at $8^{\circ}-15^{\circ} \mathrm{C}, 23^{\circ}-27^{\circ} \mathrm{C}$, and $30^{\circ}$ $35^{\circ} \mathrm{C}$. The sea water used thoughout was artificial, consisting of Neptune Salts (Westchester Aquarium Supply Co., White Plains, N.Y.) or in later experiments Instant Ocean (Aquarium Systems, Inc., Wickliffe, Ohio) made up in distilled water to a density of 1.025-1.027 and filtered through glass wool. Solutions of betaine, 
TMO, and glutamic acid were used unadjusted or adjusted to $\mathrm{pH}$ 7.5-7.8 with $\mathrm{NaOH}$.

The experiments were run at room temperature, which varied from $23^{\circ}-27^{\circ} \mathrm{C}$. Solutions other than warm and cold sea water were used at room temperature.

\section{Results}

\section{A. Morphology}

The chela comprises two segments: the larsus or movable digit and the index or fixed digit, which is an extension of the tibia (Snodgrass, 1952). The tarsus and the tibial index are similar with respect to gross structure, sensilla structure, and innervation. The following descriptions apply to both segments except where noted.

The opposed grasping surfaces of the tarsus and the index are single ridges or grasping pads of relatively pliable, unsclerotized cuticle. The ridge is round and very pliable at the proximal end of the chela, but becomes more peaked and firm distally. The pad and the tip of a digit contain only channel sensilla. The rounded back and sides of a digit, away from the pad, contain both channel and peg sensilla, although regions adjacent to the pad are nearly devoid of sensilla. Details of sensilla structure, numbers, and distribution are given elsewhere (Wyse, 1967).

The chela is supplied by two nerves, the large and small leg nerves. The small leg nerve ends at the base of the apodeme of the tarsus opener, in a proprioceptor organ containing a group of about 40 large cell bodies (O'Tanyi and Barber, 1966; Wyse, 1967). The large leg nerve innervates all of the sensory structures of the tarsus and index. Its fiber composition has been examined by light and electron microscopy (Wyse, 1967). At the distal end of the patella it contains $6-8$ presumed motor fibers $27-37 \mu$ in diameter. The remainder of the nerve is composed of presumed sensory fibers $0.12 \mu$ to $20 \mu$ in diameter, most of which are in the $0.4-2 \mu$ size range. The larger axons are individually ensheathed, but small axons occur in bundles with a mean of just over 100 axons per bundle. The nerve contains approximatly 700 isolated fibers and 3400 bundles of fibers, giving a total of 300000 to 400000 fibers in the large leg nerve.

\section{B. Responses in the Large Leg Nerve}

Responses to Mechanical Stimulation. Mechanoreceptors responding to stimulation of the pad were more convenient to study than units responding to stimulation of the back and sides of the index; they were therefore more thoroughly examined. The pad mechanoreceptors did not respond to forces applied to the finger by means of pins through the side of the chela below the pad. This control and the localized response fields (see below) indicate that the receptor terminals were located at or near the pad, rather 


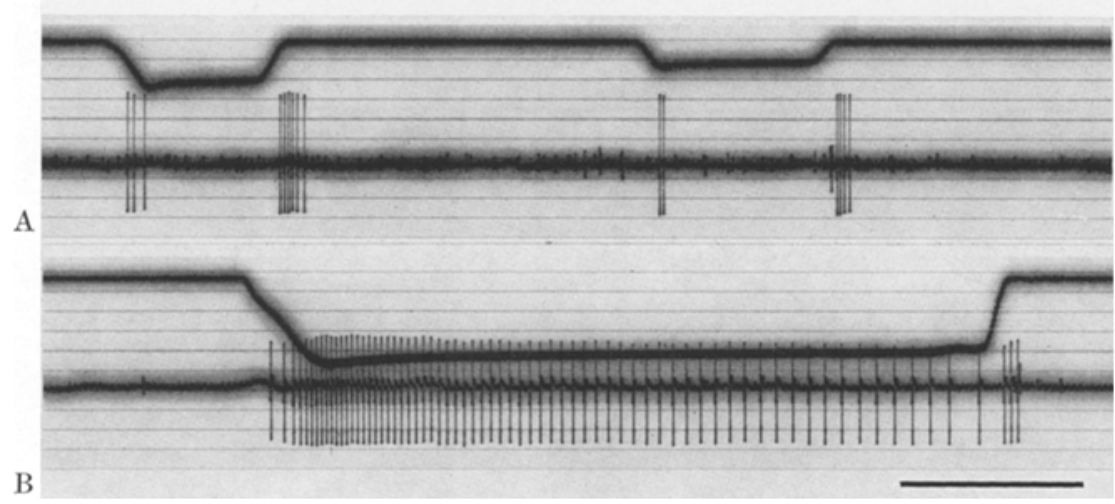

Fig. 1 A and B. Responses of pad mechanoreceptors to applied force. A Type 1 unit. Note phasic response at onset of force (downward deflection of upper beam). This unit also responded to cessation of force. B Type 2 unit. Note maintained discharge during sustained force and short burst at cessation of force. Time mark $0.5 \mathrm{sec}$

than being located at a distance and responding to general cuticular strains resulting from distant stimuli.

Pad mechanoreceptors fell into two groups : those giving rapidly adapting responses to changes of force (Type 1 ) and those giving slowly adapting responses (Type 2). Both types of mechanoreceptors had large spike amplitudes. The Type 1 units were characteristically the larger of the two and were usually the largest units in a recording situation. Examples of the two types are shown in Fig. 1. The stimulus waveform used was trapezoidal and consisted of three parts: (a) a nearly linear increase in force from zero, (b) a plateau of sustained force, and (c) a nearly linear decrease in force to zero. Because of backlash of the manipulator, the force was not constant during the plateau, but decreased with time by as much as $10 \%$. This decrease was not corrected in the analysis. Stimulation through the speaker coil, which had no backlash, gave similar responses.

Type 1 Receptors gave a burst of 1-14 spikes during increase of force. The burst usually ceased abruptly within 0.1 sec after the end of force change. At large forces some units exhibited an after-discharge upon cessation of change of force, with a break between the initial burst and the after-discharge. About half of the Type 1 receptors responded to decrease as well as to increase of force (on-off units). Responses of on-off units to cessation of force were similar to or greater than the responses to onset of force. No pure off-units were observed. Although Type 1 receptors responded to changes in downward or sideward force or pressure on the pad cuticle, they appeared more responsive to sliding stimulation, either along or across the chela axis. 


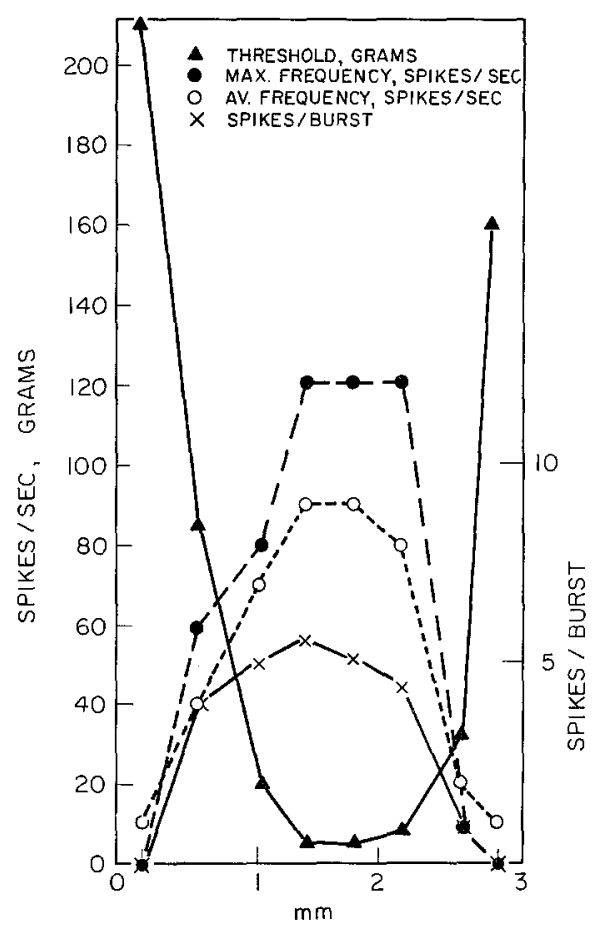

Fig. 2. Four response parameters of a Type 1 on-unit as a function of distance along the pad. The number of spikes, maximum frequency, and average frequency of a burst all have maxima corresponding to the minimum threshold of the unit. Width of stimulating probe $=1.6 \mathrm{~mm}$; distances were measured from center to center of the probe

The receptive field of a Type 1 unit with respect to direct pressure, as determined by four parameters of the responses, is shown in Fig. 2. The width of the stimulating probe was $1.6 \mathrm{~mm}$. The unit, an on-unit, responded to stimulation of only a $3 \mathrm{~mm}$ portion of the $20 \mathrm{~mm}$ pad; its threshold decreased from over $150 \mathrm{~g}$ at the edges of the field to about $6 \mathrm{~g}$ near the center. The average frequency, maximum frequency, and number of spikes in response to comparable stimuli varied inversely with the threshold at different locations. Agreement among the four parameters was fairly close. In the other cases shown fields are mapped according to variation in threshold with location. The receptive fields of other units stimulated with the $1.6 \mathrm{~mm}$ probe varied from 2.0 to $3.5 \mathrm{~mm}$. Minimum thresholds varied from 7 to $31 \mathrm{~g}$.

Since the stimulating probe was large relative to the responsive fields in the above experiments, measurements of thresholds and fields were also 

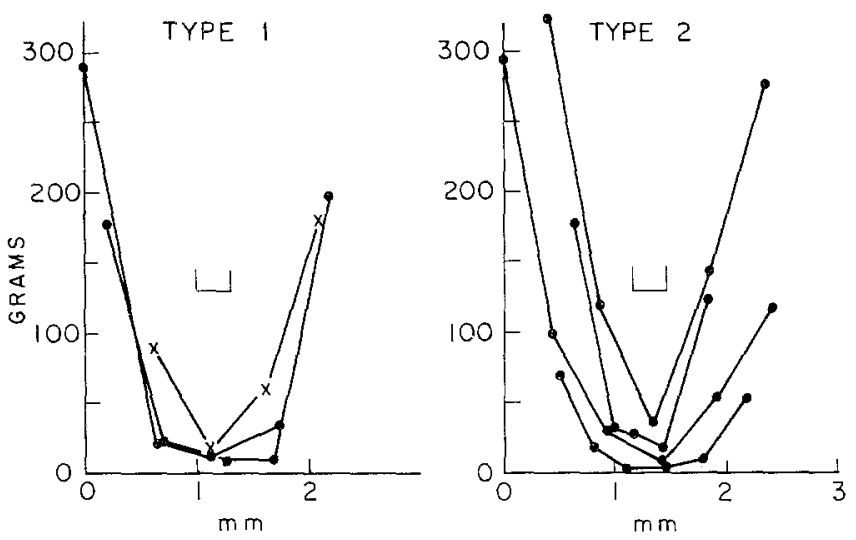

Fig. 3. Receptive fields of pad mechanoreceptors. The thresholds of seven units are plotted as a function of distance along the pad. One of the Type 1 units (crosses) was an on-unit; the others were on-off units. Inset: probe width $=0.3 \mathrm{~mm}$

conducted with a smaller probe, $0.3 \mathrm{~mm}$ in width (Fig. 3). The mapped fields varied from 1.5 to $2.2 \mathrm{~mm}$ and minimum thresholds from 9 to $18 \mathrm{~g}$. Fields obtained with the smaller probe were clearly larger than twice the probe width; therefore the responses were not due to direct contact stimulation of a punctate receptor terminal.

The spike frequencies of Type 1 units (average frequency for a burst) increased with increasing rate of force change (Fig. 4). The number of spikes per burst increased irregularly with increasing amount of change (Fig. 5). Type 1 units fatigued with repeated stimulation, responding to successive similar stimuli at about 3 sec intervals with fewer spikes and lower frequencies. The term fatigue is used here to mean decreased response to repeated stimuli, as opposed to adaptation to a single, prolonged stimulus. Fatigue further implies relatively slow recovery but as used here does not imply overstimulation. This fatigue affected the results obtained when forces and rates of change were varied. In Fig. 5, for example, the open circles correspond to responses to the last five stimuli of a series and presumably represent a fatigued state; the responses contained fewer spikes than responses to comparable early stimuli.

Since systematic variation of movements of the hand-controlled stimulator were difficult, the stimulus-response relationship of Type 1 receptors was not further examined. The available evidence indicates that Type 1 receptors are phasic and respond only to changes of force. They may code both rate and amount of force change, the former in terms of impulse frequency and the latter in terms of number of impulses. 


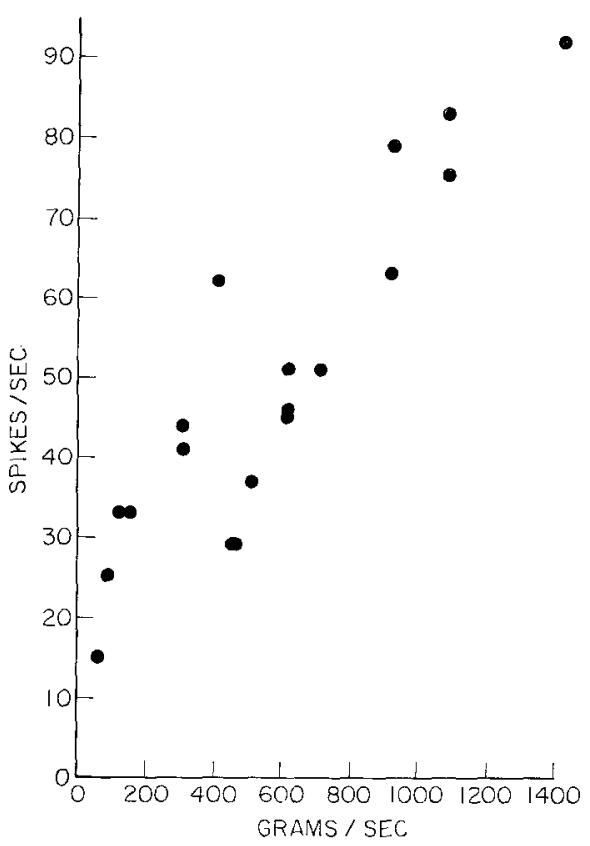

Fig. 4. Average response frequency per burst of a Type 1 unit, as a function of rate of force increase from zero. Stimuli of varying magnitude were presented in random order. Response frequency increases with increasing rate of change
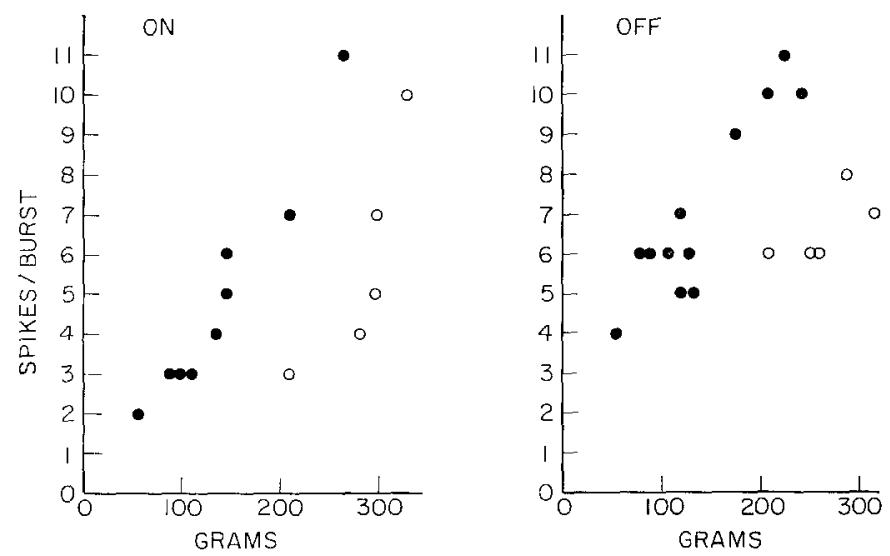

Fig. 5. Number of spikes per burst of a single Type 1 unit as a function of amount of applied force. On Responses to increase of force from zero. Off Responses to decrease of force from sustained level to zero. Three stimuli were below the velocity threshold of the unit and elicited no on-responses. Open circles are responses to the last five stimuli of the series and show the effect of fatigue. Stimuli of varying amplitude were presented in irregular order. Total duration of the series was about 


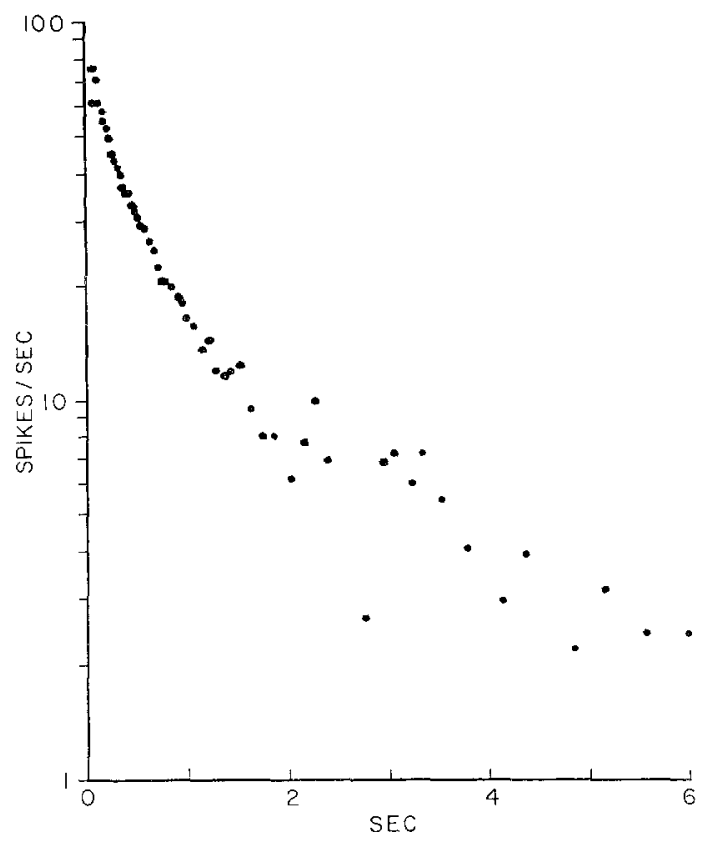

Fig. 6. Response of a Type 2 mechanoreceptor to sustained force delivered through a speaker coil. Frequency (logarithmic scale) as a function of time after onset of stimulation. The frequency decreased regularly but non-exponentially

Type 2 Mechanoreceptor responses began during increases of force and continued after the change ceased (Fig. 1B). Responses to sustained force lasted up to $14 \mathrm{sec}$, the frequency decreasing regularly but not exponentially from an initial peak (Fig. 6). Most of the Type 2 units gave a short burst of 1-10 impulses at the cessation of a stimulus. The number of spikes in such a burst increased with increasing stimulus force.

The adequate stimulus for Type 2 receptors appeared to be downward (from the direction of the opposing digit) or sideward force. Thresholds and receptive fields of 'Type 2 units were similar to those of Type 1 units, within the precision of the stimulating system (Fig. 3). Mapped fields of Type 2 units stimulated with the $0.3 \mathrm{~mm}$ probe varied from 1.2 to $2.5 \mathrm{~mm}$ with thresholds from 3 to $37 \mathrm{~g}$. As in the case of Type 1 units, Type 2 mechanoreceptors fatigued with repeated strong stimulation. Response duration was decreased more than was average frequency. Depending on the degree of fatigue, the units largely recovered their initial responses in 5 to $60 \mathrm{sec}$. Recovery rates for Type 1 units were not determined. 


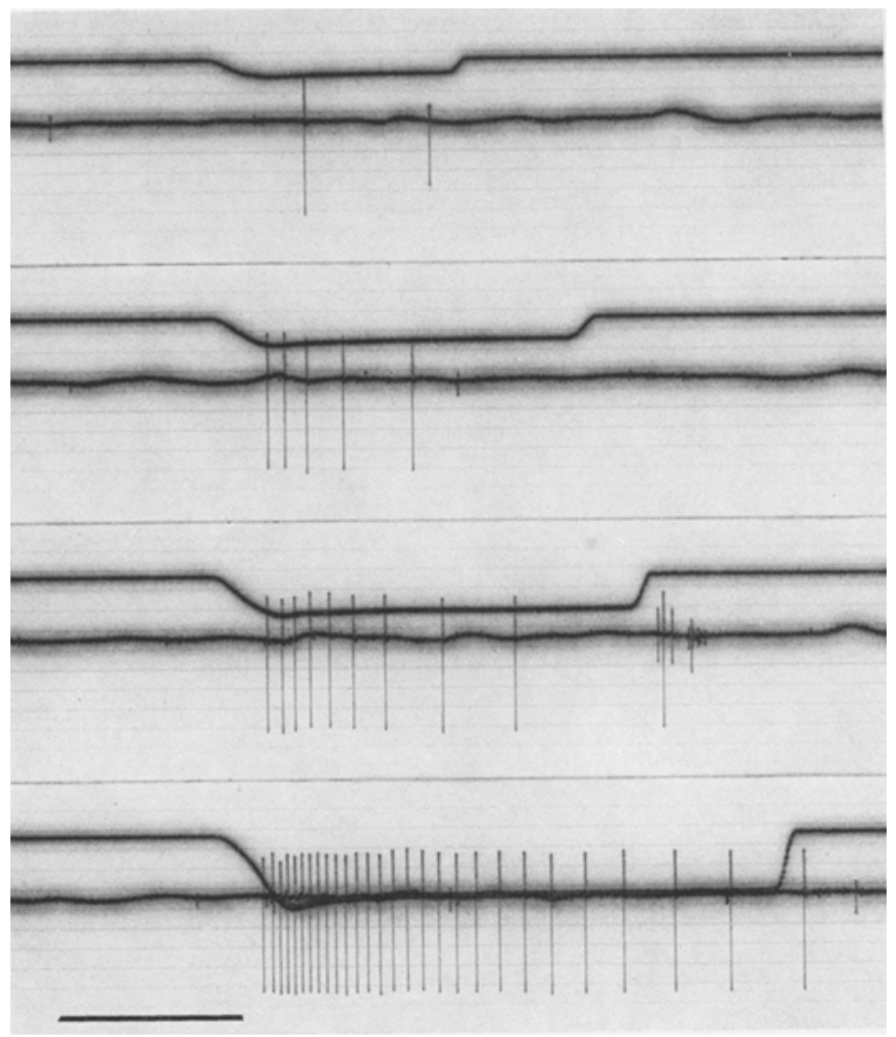

Fig. 7. Effect of increase of stimulus strength on a Type 2 unit. Stimulus forces: $34,56,77$, and $142 \mathrm{~g}$. Note increase in both frequency and duration of response with inereasing force. Time mark $0.5 \mathrm{sec}$

Both spike frequency and duration of response of Type 2 receptors increased with increasing force (Fig. 7). The initial frequency was also affected by rate of change of force, increasing with increasing rate.

The effect of stimulus force on response frequency at different locations along the pad is shown in Fig. 8. As for all units, the force-frequency relationships were similar at the three locations near the center of the field, while at the edges of the field, thresholds were higher and maximum response frequencies were lower.

Most of the mechanoreceptors were clearly either Type 1 or Type 2 units. Of 154 units responding to defined mechanical stimulation, 94 were Type 1, 45 were Type 2 , and 15 had intermediate properties. Of these 15 intermediate units, four resembled Type $I$ units but had a more pronoun- 


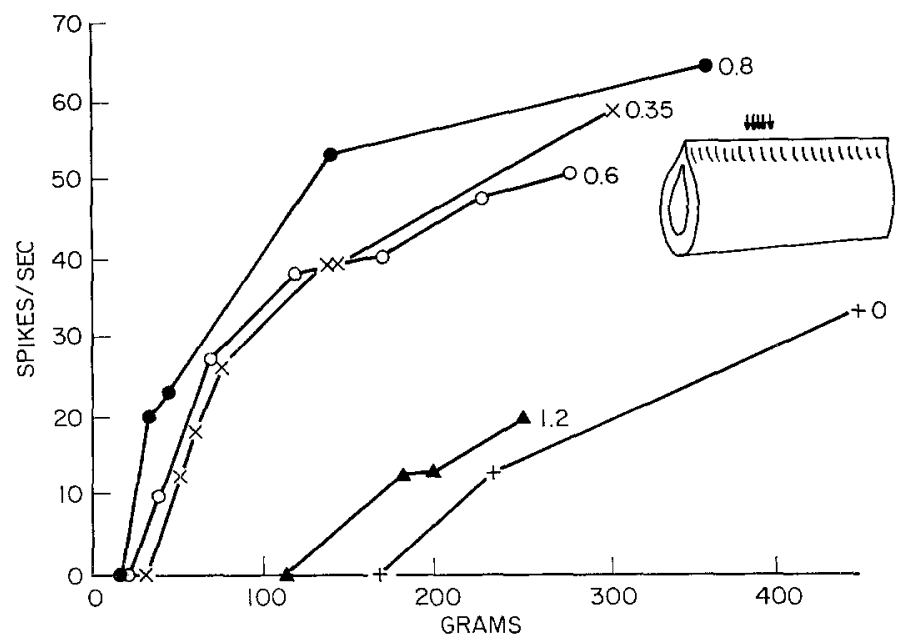

Fig. 8. Response frequency as a function of stimulus force at different places in the receptive field of a Type 2 unit. Numbers $(0,0.35,0.6,0.8$, and 1.2$)$ are distances of points stimulated in $\mathrm{mm}$ from one edge of the field. Frequency is the average for first $220 \mathrm{msec}$ of response

ced after-discharge of as many as eight spikes lasting up to $320 \mathrm{msec}$. These units exhibited a discontinuity between the transient response and the after-discharge, in contrast to the Type 2 units, in which the transistent response graded smoothly into the response to sustained force (see Fig. 1). The responses of the remaining 11 units were continuous and intermediate in duration, lasting up to $150-300 \mathrm{msec}$ after cessation of force change.

The pad mechanoreceptors are probably stimulated in nature by forces resulting from active closing of the chela. The magnitude of force produced by active closing, in response to electrical stimulation of the large leg nerve, was determined in two isolated legs. The forces produced during a tetanic contraction, recorded at the tips of the slightly opened chelae, were 170 to $220 \mathrm{~g}$. The force at a given locus is inversely proportional to its distance from the pivot. Since the tip of the chela is about five times as far from the pivot as is the pad at the base of the chela, the maximum forces on the pad due to active closing would vary from about $200 \mathrm{~g}$ at the tip to about $1000 \mathrm{~g}$ at the pad at the base of the chela. Therefore the observed thresholds of $3-40 \mathrm{~g}$ are not unreasonably high, and the range of stimulus forces used (up to $350 \mathrm{~g}$, with most stimuli below $150 \mathrm{~g}$ ) was within the range of forces to which the receptors might be exposed in nature. 
Several methods were used to determine location of pad mechanoreceptors. Shaving off the apex of the pad or even shaving off nearly all of the pad cuticle did not abolish the responses to subsequent stimuli, but in some cases slightly increased the spike frequency of the responses. This effect indicates that at least some of the mechanosensitive regions of the neurons are deep in the cuticle or below it. The possibility that dendrites of mechanoreceptors run through the cuticle cannot, however, be ruled out. Cohen (1963) found that crushing the dendrites of proprioceptive cells in the crab leg myochordotonal organ did not always abolish the responses, suggesting that mechanosensitive regions may occur near the cell bodies. A similar condition could occur in Limulus, with the cuticular sensilla of the chela pad having mechanosensitive regions located beneath the cuticle. It is more likely, however, that the mechanoreceptor dendrites are located beneath the pad, perhaps corresponding to subcuticular multipolar cells observed there by light microscopy.

The possible involvement of the multipolar cells was further explored by making transverse cuts completely through the pad, dividing the receptive field of a single unit, and then stimulating both portions of the field. In a few cases following cuts near the center of a field, the responses to stimulation on both sides of the cut were undiminished. Since such results seem to require mechanosensory elements of the same cell on both sides of the cut, these cases suggest that multipolar cells are involved. Transmission of distortion across the cut or around it (through the region adjacent to the pad) was possible, but the responses almost certainly would have been diminished.

Mechanoreceptors on the back and sides of the chela were not thoroughly studied. Stimulation of the side elicited responses characteristic of both Type 1 and Type 2 units. These units were not clearly distinguishable from pad mechanoreceptors except by location. Type I units were much more prevalent and were more responsive to sliding stimulation than to applied force. The units had apparent thresholds of $11-56 \mathrm{~g}$ and localized receptive fields of $1-3 \mathrm{~mm}$.

In three preparations the conduction velocity of mechanoreceptor fibers was determined for comparison with the compound action potential of the large leg nerve (see below). A histogram of the velocities of spikes from 81 fibers is shown in Fig. 9. The range is from 3.0 to $5.5 \mathrm{~m} / \mathrm{sec}$, and the median is $3.8 \mathrm{~m} / \mathrm{sec}$. The sample is biased toward fibers with large spikes (and high conduction velocities). It is clear, however, that at least some fibers from mechanoreceptors have relatively high conduction velocities.

Responses to Chemical Stimulation. Chemical stimulation consisted either of application of drops of solution to a chela held in air, or of exchange of solutions around a constantly immersed index or tarsus. Re- 

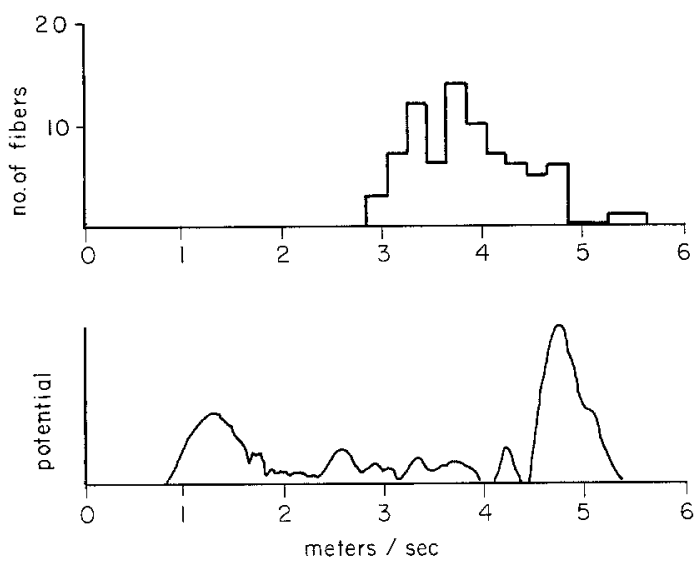

Fig. 9. Top: conduction velocities for 81 mechanoreceptor fibers. Bottom: conduction velocities of fiber populations in the large leg nerve, determined from compound action potentials. The mechanoreceptors correspond to relatively fast fibers in the nerve.

sponses recorded from chelae held in air usually involved slowly adapting activity of many very small units. Phasic responses occurred less frequently. Single fiber responses were rarely obtained, and in most cases it was impossible to analyze the activity of individual units. The time courses of two single unit responses are shown in Fig. 10.

Local stimulation with small pieces of clam or fish or with cotton swabs soaked in clam extract, fish extract, or betaine sometimes elicited chemoreceptor responses. Stimulation of the pad and tip areas gave clear responses, while stimulation of the sides of the chela was less clearly different from the control (swabs soaked in sea water). Thus some of the receptors responding to chemical stimulation are apparently located at the pad and at the chela tips.

Chemoreceptor activity was also obtained from chelae kept constantly immersed in fluid. Fig. 11 shows the responses of several units to clam extract and to glycine, but not to sea water. The responses to clam extract included several large, rapidly adapting and many small, slowly adapting units. Such activity was found in most of the bundles of favorable preparations. Activity of the small units persisted for over twenty seconds or until the stimulating fluid was washed away. The responses to glycine were of shorter duration and adapted to background level within 1-4 sec despite the constant flow of glycine. Both glycine and clam extract responses diminished with repeated stimulation. Some of the large rapidly adapting units appeared to respond in a similar fashion to both solutions, 

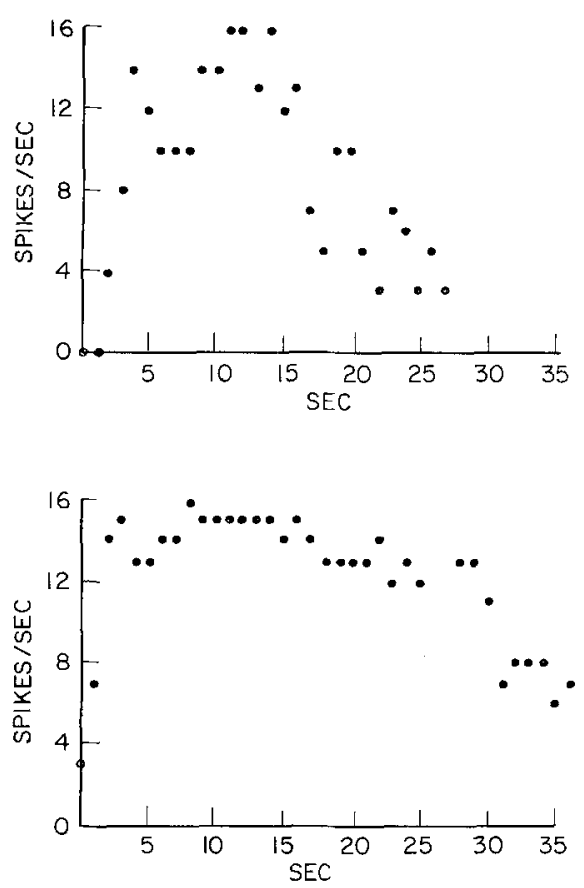

Fig. 10. Time courses of responses of two chemoreceptor units in different preparations to $0.1 \mathrm{M}$ betaine. Drop stimulation. Open circles at time 0 indicate pre-stimulus frequencies.

although positive identification is difficult. At least some of the small slowly adapting units responding to clam extract did not respond to glycine.

Since rapidly adapting chemoreceptor responses occurred with constant fluid flow as well as with drops of solution, the rapid adaptation to certain chemicals is presumably an intrinsic receptor property.

The following chemicals elicited chemoreceptor responses: clam extract, fish extract, betaine, glycine, glutamic acid, and trimethylamine oxide. Isotonic sucrose, mixtures of isotonic sucrose and sea water, and sea water at $p H$ 2-9 were not effective. Sea water at $p H 10$ was sometimes weakly stimulatory. Apparently single units sometimes responded to more than one solution, including the following combinations: betaine and fish extract, glycine and clam extract, and betaine and distilled water.

Behavioral Responses to Chemical Stimulation. In intact animals, application of chemical stimuli to the chela usually elicited a transient opening 


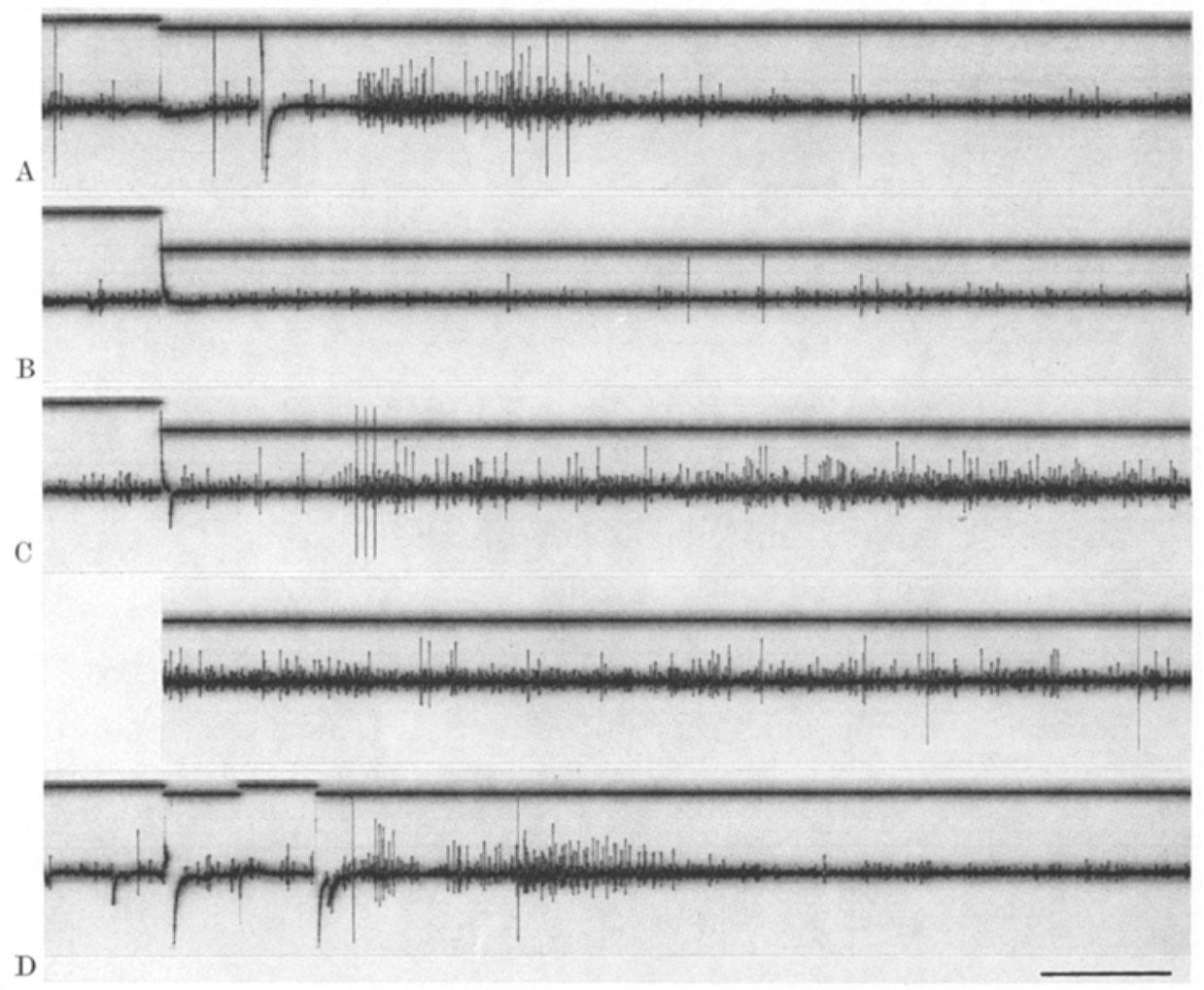

Fig. $11 \mathrm{~A}-\mathrm{D}$. Chemoreceptor responses during continuous flow of stimulating solutions. Upper beam in all records gives onset of fluid exchange and indicates the fluid used. Rate of fluid exchange: $50 \%$ in about 1.2 sec, $95 \%$ in about 4 sec. Spikes of largest unit retouched. A Response to $0.1 \mathrm{M}$ glycine. Note rapid adaptation. B No response to sea water. C Response to clam extract; the record is continued on the following line. Response persisted for over 20 sec, then ceased after switching to sea water. D Response to glycine, similar to top record. Time mark $0.5 \mathrm{sec}$

response. The chela was rapidly opened and sometimes held open for several seconds, slowly relaxed to a partly open position, and sometimes oscillated around this position. The final return to rest position was usually slow. Similar application of sea water elicited a shorter and weaker opening or no response at all. Several preparations showed clear and repeatable responses to betaine (Fig. 12) or to fish juice. Trimethylamine oxide was less clearly stimulatory. There was usually no response to sea water before chemical stimulation, but after chemical stimulation sea water evoked a small response that diminished rapidly with repeated sea 
A
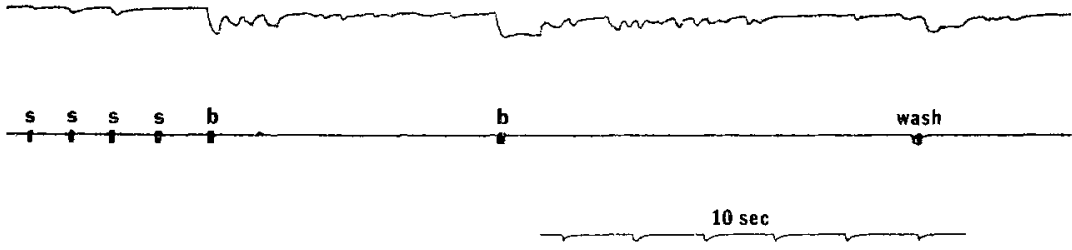

$\mathbf{B}$

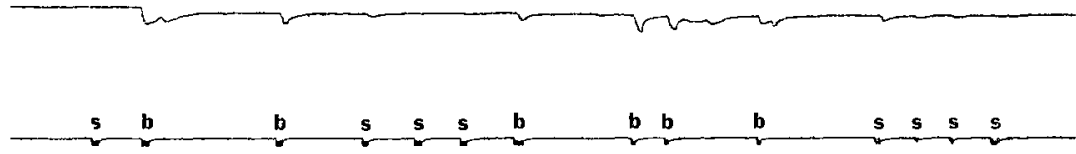

Fig. $12 \mathrm{~A}$ and B. Behavioral responses to chemical stimulation. A and B Two portions of a kymograph record, separated by about $6 \mathrm{~min}$. First and fourth traces: tarsal movement response (downward $=$ opening). Second and fifth traces: marker for tibial stimulation with sea water $(s)$ or betaine (0.1M) (b). "Wash" indicates prolonged washing with sea water. Third trace: Time marker. Intervals, $10 \mathrm{sec}$

water application. Such responses were probably due to residual chemical solution on the chela, rather than to the sea water itself.

Chemoreceptors of the chela appear to initiate feeding behavior in intact animals in aquaria. When a chela touches a piece of fish the animal moves toward it and the other legs change from walking position (legs held laterally) to shoveling movements directed toward the mouth. Contact of the fish with the margin of the cephalothorax, abdomen, or gills is not effective.

Responses to Water and to Dilute Sea Water. Over half of the dissected bundles tested contained units responding to stimulation of the chela with distilled water. Of these units fewer than half also responded to $40 \%$ sea water and very few to $60 \%$ and $80 \%$ sea water. Hyperosmotic solutions were not tried. Units responding to distilled water, but not to $40 \%$ sea water, were found in chelae constantly immersed in fluid.

The responses to hypoosmotic solutions consisted of regular or irregular tonic activity of medium-sized units. The responses to water usually persisted until the chela was washed with sea water, but responses to dilute sea water usually lasted only 5-10 sec. The responses always ceased after application of sea water and were usually repeatable. Direct application of water to the nerve exposed for recording did not induce comparable responses in the three preparations tested.

The question of whether responses to water and to dilute sea water were due to low osmotic concentration or low ionic strength was not completely answered. On some occasions the same or similar units responded to application of both $40 \%$ sea water and $40 \%$ sea water made iso- 
tonic with $1 \mathrm{M}$ sucrose, but not to $100 \%$ sea water. Most responses to $40 \%$ sea water in sucrose or to isotonic sucrose were weaker than the responses to $40 \%$ sea water or to distilled water. Thus both ionic strength and osmotic concentration may have been involved.

Responses to Thermal Stimulation. Two kinds of responses to warm $\left(30-35^{\circ} \mathrm{C}\right.$ ) sea water occurred. (Air temperatures around the chelae were $23-25^{\circ} \mathrm{C}$.) Phasic responses from large and medium amplitude units were common, and usually adapted on repeated stimulation. Some units responding to warm sea water stimulation also responded to mechanical stimulation. Tonic responses of moderate amplitude units to warm sea water occurred less frequently.

Cold sea water $\left(8-10^{\circ} \mathrm{C}\right)$ evoked both phasic and tonic responses. The phasic units had large or medium amplitudes. Tonic units had medium amplitudes and usually had long (1-3 sec) latencies. In two cases individual tonic units were found which responded to warm and to cold sea water, but not to sea water at room temperature.

Following stimulation with cold sea water, phasic responses to room temperature sea water sometimes resembled weak warm responses, and following warm stimulation, room temperature responses either resembled weak cold responses or disappeared. Thus the phasic temperature receptors seem to respond to temperature change rather than to absolute temperature.

Conduction Velocities and Fiber Populations in the Large Nerve. In order to relate the observed receptor responses to the anatomy of the large leg nerve, the compound action potential of the whole nerve was recorded. The portion of the nerve in the patella and femur was isolated and placed over seven pairs of silver wires in a moist chamber. The compound action potential consisted of two major peaks, one fast and one slow, and several minor intermediate peaks. Fig. 9 shows an example, rescaled in units of conduction velocity. Fibers of all velocities were seen, and the peaks corresponded to only the relatively well represented velocity classes. The large fast peak corresponds to a mean conduction velocity of about $4.7 \mathrm{~m} / \mathrm{sec}$, the major intermediate peaks to $3.4,2.5$, and $1.8 \mathrm{~m} / \mathrm{sec}$, and the large slow peak to about $1.2 \mathrm{~m} / \mathrm{sec}$. The large fast peak presumably includes the 6-8 large motor fibers present at this level. It must include other elements, however, because at least 15 quantal increases, each a separate fiber, have been counted in the first peak, after which the contribution of each new spike became too small to be noted. Furthermore a similar large fast peak was recorded from nerve branches in the tibia, at a level where only sensory fibers are present. Thus the large fast peak contains fast sensory fibers as well as the motor fibers. The rapidly conducting mechanoreceptor fibers (Fig. 9) are included in the faster peaks and may comprise a major portion of them. 


\section{Responses in the Small Leg Nerve}

All units observed in the small leg nerve were proprioceptors of the tibiotarsal joint, with properties similar to the units described by O'Tanyi and Barber (1966). The firing frequency of active units always increased with depression (closing) of the tarsus and decreased or became silent with elevation (opening). Sustained depression caused a transient burst of large impulses and sustained firing of smaller units. The large phasic units adapted completely within $0.25 \mathrm{sec}$, while the smaller tonic units continued to respond for several mintes. Increased depression of the tarsus increased both the firing frequency of active units and the number of active units. At the cessation of depression there was a silent period of 0.1-0.2 sec, after which the resting activity of the tonic units resumed. No units responding to opening or to open position were seen in over 30 preparations.

Aside from movement and position changes of the tarsus, the only modification of the resting discharge of proprioceptive units in the intact chela was caused by probing at the tibiotarsal articular membrane. Increased firing frequency resulted, usually in smaller units. Application of other stimuli resulted in activity only when pseudoreflex closing of the tarsus occurred (see Hoyle, 1958). Of the stimuli tested in this study, therefore, the small leg nerve mediated proprioception only.

\section{Diseussion}

\section{A. Functional Characteristics of the Receptors}

Mechanoreceptors. The two types of mechanoreceptors of the chela pad differ primarily in rate of adaptation to sustained stimulus force. Type 1 mechanoreceptors are velocity sensitive, firing when the stimulus is changing, whereas Type 2 units are pressure sensitive and give a repetitive discharge during static deformation. Impulse frequency of Type 1 units increases with increasing rate of force change, while frequency of Type 2 units increases primarily with increasing amount of force, although the initial discharge is also velocity sensitive. Such velocity sensitivity is a corollary of any adaptation of a response to a sustained stimulus.

The mechanoreceptors are apparently located near the inner cuticular surface and are stimulated by transmission of distortion through the relatively pliable cuticle. In this respect they are analogous to the epidermal mechanoreceptors of the abdomen of hermit crabs (Chapple, 1966). The $100 \mu$ layer of pad cuticle between the stimulus and the receptors probably alters the stimulus properties considerably, receptor thresholds being raised by dissipation of distortion. The adaptation of the units may result in part from mechanical low-frequency filtration of the kind (but not the degree) found in the Pacinian corpuscle (Loewenstein and Skalak, 1966). 
The fatigue of units to repeated stimulation may result from slow recovery or inelasticity of the pad cuticle. It is likely that one or both of the mechanoreceptor types correspond to multipolar cells under the pad. These cells lie in the required locus under the pad and their large axons are consistent with the large spike amplitudes and rapid conduction velocities of mechanoreceptor units. Their branched distal processes, extending $300-500 \mu$ along the pad axis, may explain the occasional finding of responses of a unit persisting on both sides of a transverse cut.

Chemoreceptors. Most responses to chemical stimulation are tonic and slowly adapting, as has been observed in other arthropods. The phasic responses seen with both drop stimulation and continuous immersion are less common, although phasic chemoreceptor responses to drop stimulation occur in Panulirus (Laverack, 1964).

The clearest chemoreceptor responses were seen with stimulus concentrations of $0.01-0.1 \mathrm{M}$. Less clear responses were sometimes seen at lower concentrations. The gnathobase chemoreceptors also have high thresholds for amino acids. Barber (1961 a, b) and Barber and Hayes (1963) found that $0.5 \mathrm{M}$ solutions of amino acids were required to give responses approaching those to clam extract. The only threshold they reported (for one unit to glycine) was between 0.01 and $0.001 \mathrm{M}$. In other aquatic arthropods concentrations of amino acids and amines usually used to demonstrate chemoreceptor activity range from $0.001 \mathrm{M}$ (Case and Gwilliam, 1961 ; Levandowsky and Hodgson, 1965) to 0.05M (Case, 1964), $0.1 \mathrm{M}$ (Laverack, 1963, 1964) and $0.25 \mathrm{M}$ (Hodgson, 1958). The lowest threshold reported (Case and Gwilliam, 1961) was $5 \times 10^{-5} \mathrm{M}$ for Carcinus receptors to glutamic acid. It is likely, therefore, that in Limulus as in other arthropods studied the receptors may not be extremely sensitive to these chemicals and may function for only short-range detection of food.

The use of chemoreceptors of the chelae for short-range food detection is indicted by behavioral evidence. Contact of pieces of food with the chelae initiates feeding behavior in aquaria. In nature, Limulus is said to feed primarily on annelids and molluses, by picking them up with the chelae and transferring them to the gnathobases (Shipley, 1909). Whether these chemoreceptors are used in distance as well as contact chemoreception is not known; behavioral changes after pieces of fish are placed in the aquarium indicate that distance chemoreceptors are present (personal observation). The chemoreceptors may interact with pad mechanoreceptors in feeding, since contact with pieces of food elicits grasping and movement of the chelae to the gnathobases, whilefluids alone elicit chela opening.

Previous studies of chemoreceptor responses in arthropods have not employed constant fluid immersion. Therefore response variation has been difficult to separate from stimulus variation. Although in this study there 
was no marked difference in responses to drop stimulation in air and to constant immersion stimulation, the latter affords a degree of reliability that is not available with the techniques (drop stimulation of receptors in air, alternate filling and draining of a chamber) previously employed. With stimulation other than constant immersion, the significance of rapidly adapting chemoreceptor responses would be difficult to evaluate.

The chemoreceptors localized to the pad and tips of the digits probably correspond to the channel sensilla found there; channel sensilla are the only sensory structures in these areas that could possibly be open to the outside.

Water Receptors. Osmoreceptors are presumed to exist in marine arthropods but have not been identified (Bullock and Horridge, 1965). Responses have been recorded from Limulus gnathobase receptors upon application of distilled water (Barber and Hayes, 1963) and from motor nerves of the lobster Jasus in response to stimulation of the antennules with 75-80\% sea water (Krijgsman and Krijgsman, 1954).

Apparent thresholds to dilute sea water stimulation of the chela in air vary from below $40 \%$ to above $80 \%$ sea water. The concentrations at the receptor surface may have been higher due to mixing with residual sea water on the chela. Whether these receptors function in nature as monitors of dilution of sea water by fresh water is not resolved. Distilled water certainly constitutes a non-physiological stimulus, but the dilute sea water solutions are within the range encountered by a coastal animal such as Limulus (MoManus, 1969; Robertson, 1970).

Thermoreceptors. Most of the responses to warm sea water are phasic, apparently responding to temperature change rather than to absolute temperature. However, some other units are only partially adapting and seem to be responsive to absolute temperature. The sudden temperature changes used in stimulation may not be common in the natural environment of Limulus, but presumably would occur at thermoclines and on entering and leaving the water at breeding. Murray (1962) noted that most sense organs are temperature sensitive and maintained that temperature receptors in the restricted sense must be differentiated from temperature-sensitive sense organs that are not utilized as such by the animal, and from temperature-sensitive nociceptors. Absolute temperature sensitivity of the chela units has not been quantified. The temperature changes used were pronounced (about $6-16^{\circ} \mathrm{C}$ ) but response differences were also great. The identification of these units as temperature receptors is provisional; definite identification requires more evidence of sensitivity and of behavioral responses to thermal stimulation.

Proprioceptors. The proprioceptive units of the tibio-tarsal joint (tarso-dactylar joint, Pringle, 1956) fall into two classes. Each unit has 
either a phasic response to elosing movement or a tonic response to closed position. The tonic units are somewhat movement sensitive, however, in that they exhibit silent periods after opening.

Known proprioceptive organs of arthropod appendages usually contain both flexion-sensitive and extension-sensitive units (Pringle, 1956; Barber, 1960; Rathmeyer, 1967; Wyse and Maynard, 1965; crab leg studies reviewed in Bush, 1965). Two of the organs found in crab legs may be entirely relaxation sensitive (CP2 and MC2, Bush, 1965), but other organs with stretch-sensitive units are also present at these joints. The tibiotarsal joint of Limulus is therefore unusual in having one extreme position (open) signaled only by the absence of proprioceptive impulses.

Under certain conditions the pad mechanoreceptors, as well as these joint proprioceptors, may provide proprioceptive information during active or forced closing of the chela. When grasping an object the pad receptors may provide a measure of force at the joint (muscle tension), and the joint receptors may give a measure of joint position. Thus metric and tonic aspects of closing could be monitored separately. This situation would necessarily occur if the force of active closing, spread over the length of the chela, is sufficient to excite the pad receptors. When an object is grasped by the chela, the relationship of force at the joint to sensory output of the pad receptors must vary with the position along the pad and the size of the object grasped. The position and size of the object are coded by the mechanoreceptors, but rather elaborate central information processing would be required for measurement of absolute closing tension under these conditions.

Relation to Gnalhobase Receptors. Many of the receptors of the chelae have properties similar to the receptors of the gnathobase spines. Barber (1956, 1961 a, b) and Barber and Hayes (1963) found responses at the gnathobases to chemical, thermal, osmotic, and mechanical stimulation. The chemoreceptors at both ends of the legs are similar in response pattern and sensitivity, as well as in presumed structure. Water receptors and warm-sensitive receptors may also be similar at the two locations, although the gnathobase units have not been described in sufficent detail to permit comparison. Cold-sensitive units have not been reported previously for Limulus. Gnathobase mechanoreceptors have not been studied, in detail. They must differ considerably in anatomical arrangement and almost certainly in properties from the pad mechanoreceptors of the chelae, described above.

\section{B. Relation of Fiber Size, Conduction Velocity, and Receptor Responses}

Microscopic evidence and conduction velocity studies show that the large leg nerve contains few large, rapidly conducting fibers and very many small slowly conducting fibers, with a rather complete spectrum of 
intermediates. Fiber size and velocity may be correlated as follows (values are for large adults about $24 \mathrm{~cm}$ wide): the highest velocity in the compound action potential $(5.3 \mathrm{~m} / \mathrm{sec})$ should represent the largest fibers (about $35 \mu$ ). If conduction velocity is proportional to the square root of fiber diameter (reviewed in Bullock and Horridge, 1965), then a velocity of $4 \mathrm{~m} / \mathrm{sec}$ corresponds to fibers of $20 \mu, 2 \mathrm{~m} / \mathrm{sec}$ to $5 \mu$, and $1 \mathrm{~m} / \mathrm{sec}$ to $1.2 \mu$. The large slow peak of the compound action potential then corresponds to the very numerous fibers of about 0.5 to $3 \mu$.

It is then of interest to attempt to correlate the size and velocity classes of the leg nerve with the receptor classes studied. To a certain extent, the extracellularly recorded spike amplitudes are a direct reflection of fiber size, with variations due to differing sizes of dissected bundles and differing distances of fibers in a bundle from the recording electrodes. Although the recorded spike amplitudes from each receptor class are variable, there are relatively consistent differences in amplitude between some of the classes. Type 1 mechanoreceptor units are characteristically larger than Type 2; phasic warm units are larger than phasic cold units. All of the above types are usually larger than the remaining classes, namely phasic chemoreceptors, warm and cold tonic receptors, water receptors, and tonic chemoreceptors, in order of decreasing average amplitude. Certain of these groups may then correspond to the peaks of decreasing conduction velocity in the compound action potential. It has been possible to test this relation directly only for the large mechanoreceptors, the velocities of which range from $3.0-5.5 \mathrm{~m} / \mathrm{sec}$, with a median of $3.8 \mathrm{~m} / \mathrm{sec}$. These values correspond to the fastest three peaks of the compound action potential (Fig. 9) and to fibers in the order of $20 \mu$.

Conduction velocities of the other receptor classes were not successfully determined. In part because of the difficulty of providing local fluid stimuli, the spikes obtained were too numerous and too small to be analysed. It can be assumed, however, that the large slow peak of the compound action potential, and thus the numerous small fibers $0.5-3 \mu$ in diameter, correspond at least in part to chemoreceptors, since nearly all tonic chemoreceptor responses are of the smallest amplitude.

\section{Importance of the Receptors of the Chelae}

The Limulus chela is characterized by a few kinds of relatively simple receptor structures, and in contrast, a relatively wide variety of physiological receptor types. For most modalities of stimulation, phasic and tonic receptors provide parallel channels, separately coding the dynamic and static aspects of a stimulus.

The importance of this array to the animal is suggested by the large numbers of sensory cells involved. There are 300000-400000 sensory fibers from each chela, or about 3000000 for the whole animal. In con- 
trast each compound eye has only about 1000 ommatidia or 12000 photoreceptor cells. The large numbers of chela sensory neurons must constitute a major portion of the total sensory input into the central nervous system.

The total number of sensory channels from the chelae of Limulus is also large in comparison to other major arthropod sensory structures such as insect antennae and crustacean antennules and legs. One antenna of a worker honey bee has about 12000 cuticular sensilla, supplied by about 90000 sensory cells (Lacher, 1964), whereas each Limulus chela has about 31000 cuticular sensilla and 300000-400000 sensory fibers (Wyse, 1967). The antenna of the silkmoth Antheraea has about 60000 sensilla but only about 150000 receptor cells (Schneider, Lacher and Kaissling, 1964), and an antennule of the spiny lobster contains 1000 1500 sensilla and 500000-600000 sensory cells (Laverack, 1964). Crayfish have 35000 fibers in the chelate leg nerve (Nunnemacher, Camougis, and McAlear, 1962) and a maximum of 20075 fibers in a walking leg nerve. Limulus may then have more primary afferent fibers than the studied insects or crustaceans.

Such a very large number of primarily chemoreceptive sensory fibers would be expected to require major central nervous structures for handling the incoming information. The corpora pedunculata (mushroom bodies) of arthropods are highly organized, paired brain structures, commonly supposed to be higher integrative centers concerned in the most complex behavior of the animals. They may serve primarily to integrate information from visual and chemosensory distance receptors (Maynard, 1967). Limulus lacks complex behavior, but has extremely large and well developed corpora pedunculata of unknown function (Hanström, 1926). Large tracts of very small fibers, apparently continuations of chemosensory fibers, run cephalad from the legs to the corpora pedunculata, probably with intervening synapses in the tritocerebral cheliceral ganglion (Patten, 1912, and personal observation). From the results of the present study, it seems possible that the enormous sensory input of 3000000 chela chemosensory fibers, plus nearly 1000000 similar fibers from the gnathobase spines (Hayes, 1966) is integrated in the corpora pedunculata, and that the numerical size of this sensory input is causally related to the tremendous development of the corpora pedunculata in the Limulus brain.

I am deeply indebted to Dr. Donald M. Maynard for his advice and encouragement. I wish to thank Drs. David R. Bentley, Michael S. Laverack, Gernot Wendler, and Richard Norman for helpful discussion and suggestions.

This work is based on part of a dissertation submitted in partial fulfillment of the requirements for the degree of Doctor of Philosophy at the University of Michigan. The work was carried out under tenure of a Cooperative Graduate Fellowship 
and a Graduate Fellowship from the National Science Foundation. Much of the equipment used was made available by the National Institutes of Health Graduate Training Grant No. 5 TI GMI 989 and by a grant from the Graduate Student Research Fund of The University of Michigan.

\section{References}

Barber, S. B.: Chemoreception and proprioception in Limulus. J. exp. Zool. 131, $51-74$ (1956).

- Structure and properties of Limulus articular proprioceptors. J. exp. Zool. 143, 283-321 (1960).

- Responses of Limulus chemoreceptors to amino acid stimulation. Amer. Zoologist 1,435 (1961 a).

- Chemoreception and thermoreception. In: The physiology of Crustacea, Vol. II (T. H. Waterman, ed.), pp. 109-131. New York: Academic Press 1961 b.

- Hayes, W. F.: Properties of Limulus chemoreceptors. Proc. XVI Int. Cong. Zool. Wash. 3, 76-78 (1963).

Bullock, T. H., Horridge, G. A.: Structure and function in the nervous systems of intervertebrates, 2 vols., pp. 1719. San Francisco: W. H. Freeman and Co. 1965.

Bush, B. M. H. : Proprioception by chordotonal organs in the mero-carpopodite and carpo-propodite joints of Carcinus maenas legs. Comp. Biochem. Physiol. 14, 185-199 (1965).

Case, J.: Properties of the dactyl chemoreceptors of Cancer antennarius Stimpson and $C$. productus Randall. Biol. Bull. 127, 428-446 (1964).

- Gwilliam, G. F.: Amino acid sensitivity of the dactyl chemoreceptors of Carcinides maenas. Biol. Bull. 121, 449-455 (1961).

Chapple, W. D.: Sensory modalities and receptive fields in the abdominal nervous system of the hermit Pagurus granosimanus (Stimpson). J. exp. Biol. 44, 209223 (1966).

Cohen, M. J.: The crustacean myochordotonal organ as a proprioceptive system. Comp. Biochem. Physiol. 8, 223-243 (1963).

Hanström, B.: Das Nervensystem und die Sinnesorgane von Limulus polyphemus. Acta Univ. Lund. Avd. 2, 22 (5), 1-79 (1926).

Hayes, W. F.: Chemoreceptor sensillum structure in Limulus. J. Morph. 119, 121 $142(1966)$.

- Fine structure of the chemoreceptor sensillum in Limulus. J. Morph. 133, 205240 (1971).

- Barber, S. B.: Proprioceptor distribution and properties in Limulus walking legs. J. exp. Zool. 165, 195-210 (1967).

Hodgson, E. S.: Electrophysiological studies of arthropod chemoreception. III. Chemoreceptors of terrestrial and freshwater arthropods. Biol. Bull. 115, 114-125 (1958).

- The chemical senses and changing viewpoints in sensory physiology. In: Viewn points in biology, (J. D. Carthy and C. L. Duddington, eds). vol. 4, p. 83-123. London: Butterworths 1965.

Hoyle, G.: Studies on neuromuscular transmission in Limulus. Biol. Bull. 115, 209-218 (1958).

Krijgsman, B. J., Krijgsman, N. E.: Osmorezeption in Jasus lalandii. Z. vergl. Physiol. 37, 78-81 (1954).

Lacher, V.: Electrophysiologische Untersuchungen an einzelnen Rezeptoren für Geruch, Kohlendioxyd, Luftfeuchtigkeit, und Temperatur auf den Antennen der Arbeitsbiene und der Drohne (Apis mellifica L.). Z. vergl. Physiol. 48, 587-623 (1964). 
Laverack, M. S.: Aspects of chemoreception in Crustacea. Comp. Biochem. Physiol. $8,141-151(1963)$.

- The antennular sense organs of Panulirus argus. Comp. Biochem. Physiol. 13, 301-321 (1964).

Levandowsky, M., Hodgson, E. S.: Amino acid and amine receptors of lobsters. Comp. Biochem. Physiol. 16, 159-161 (1965).

Loewenstein, W. R., Skalak, R.: Mechanical transmission in a Pacinian corpuscle. An analysis and a theory. J. Physiol. (Lond.) 182, 346-378 (1966).

Maynard, D. M. : Organization of central ganglia. In: Invertebrate nervous systems. Their significance for mammalian neurophysiology (C. A. G. Wiersma, ed.), pp. 231-255. Chicago: U. Chicago Press 1967.

McManus, J. J.: Osmotic relations in the horseshoe crab, Limulus polyphemus. Amer. Midl. Natur. 81, 569-573 (1969).

Murray, R. W.: Temperature receptors. In: Advances in comparative physiology and biochemistry. (0. Lowenstein, ed.), vol. 1, pp. 117-175. New York: Academic Press 1962.

Nunnemacher, R. F., Camougis, G., McAlear, J. H.: The fine structure of the crayfish nervous system. Fifth Int. Congr. Electron Microscopy. p. N 11. New York: Academic Press 1962.

O'Tanyi, T. J., Jr., Barber, S. B.: Stretch receptors in Limulus limbs. Amer. Zoologist 6, 519-520 (1966).

Patten, W.: On the morphology and physiology of the brain and sense organs of Limulus. Quart. J. micr. Sci. 35, 1-96 (1893).

- The evolution of the vertebrates and their kin, pp. 486. Philadelphia : Blakiston 1912.

Pringle, J. W. S.: Proprioception in Limulus. J. exp. Biol. 33, 658-667 (1956).

Rathmayer, W.: Electrophysiologische Untersuchungen an Proprioceptoren im Bein einer Vogelspinne (Eurypelma hentzi Chamb.) Z. vergl. Physiol. 54, 438-454 (1967).

Robertson, J. D.: Osmotic and ionic regulation in the horseshoe crab Limulus polyphemus (Linnaeus). Biol. Bull. 138, 157-183 (1970).

Schneider, D., Lacher, V., Kaissling, K. E.: Die Reaktionsweise und das Reaktionsspektrum von Riechzellen bei Antheraea pernyi (Lepidoptera, Saturniidae). Z. vergl. Physiol. 48, 632-662 (1964).

Shipley, A. E.: Introduction to Arachnida and Xiphosura. In: Cambridge natural history, (S. F. Harmer, ed.), vol, 4, pp. 253-279. New York: Macmillan 1909.

Snodgrass, R. E.: A Textbook of arthropod anatomy, pp. 363. Ithaca: Cornell U. Press 1952.

Sutherland, R.: Fine structure of the crayfish thoracic cord and leg nerves. Amer. Zoologist 6, 520 (1966).

Wyse, G. A.: Functional organization of receptors in the chelae of Limulus polyphemus. Ph.D. Dissertation. The University of Michigan 1967.

- Maynard, D. M.: Joint receptors in the antennule of Panulirus argus Latreille. J. exp. Biol. 42, 521-535 (1965).

Dr. Gordon A. Wyse

Department of Zoology

University of Massachusetts

Amherst, Mass. 01002 